\title{
Probing Ultrafast Carrier Dynamics by Laser-Combined STM
}

\author{
Hidemi Shigekawa*, Shoji Yoshida and Osamu Takeuchi \\ Faculty of pure and applied sciences, University of Tsukuba, Tsukuba, 305-8573, Japan
}

With the progress of science and technology, we are now facing limitations of current technologies that cannot be overcome by the simple expansion of existing engineering concepts. Thus, the introduction of new concepts based on new ideas, as well as the discovery of new physical properties and their applications, is a key factor in breaking this deadlock. However, for example, the technology node of semiconductor devices has become as small as $20-30 \mathrm{~nm}$, and the fluctuations in the spatial distribution of dopants, which are introduced to control the properties of devices, and in the nanostructured interface between dissimilar materials directly affect the device functions obtained. Charge transfer in composite materials governing, for example, phase transitions, photoelectric conversion and catalysis is generally over the picoseond-to-subpicosecond range. Phonon-related phenomena are much faster. Namely, with the miniaturization of functional devices consisting of composite materials, quantum dynamics, which has been analyzed by techniques providing spatially and/or temporally averaged information, does not provide a sufficiently detailed description for the analysis and design of macroscopic functions.

Therefore, the development of a method for exploring the ultrafast transient dynamics in small organized structures with high spatial resolution is a key factor for further advances in current science and technology. Scanning tunneling microscopy (STM) is one of the most promising techniques for the analysis of such properties because of its high spatial resolution. However, since its temporal resolution is low, the development of STM and related techniques with high-timeresolution has been an attractive target since its invention [1]. We have been working on lasercombined STM [1-8 and references therein], and recently we have succeeded in developing a new microscopy technique by combining STM with ultrashort-pulse laser technology, which enables the visualization of ultrafast carrier dynamics up to the femtosecond range and even at the single-atomic level [5, 7, Fig. 1]. The combination of STM with optical technology has advantages to enable the analysis of photo-induced dynamics on the nanoscale as well as the realization of ultrafast timeresolved microscopy. In OPP-STM, a non-equilibrium carrier distribution is generated using ultrashort laser pulses and its relaxation processes are probed by STM using the OPP method realized in STM.

Furthermore, with the development of a new modulation technique of circularly polarized light, detection of spin dynamics has been realized [9]. We have succeeded in observing spin dynamics in D'yakonov-Perel mechanism. The relaxation of spins optically oriented in single quantum wells formed by GaAs/AlGaAs was observed independently (Fig. 2). We have also succeeded in observing spin precession dynamics (Fig. 3). The spin precession in GaAs under magnetic field was successfully probed using tunneling current by the time-resolved STM. Because the nanoscale properties of materials differ from bulk structures due to various factors, the successive development of novel microscopy techniques based on new ideas and the complementary use of these microscopy techniques will greatly aid the further development of nanoscale science and technology [10].

* http://dora.bk.tsukuba.ac.jp/, e-mail: hidemi@ims.tsukuba.ac.jp 
References:

[1] Y. Terada, S. Yoshida, O. Takeuchi, and H. Shigekawa: J. Phys.: Condensed Matter 22 (2010) 264008.

[2] Y. Terada, S. Yoshida, and H. Shigekawa: Advances in Optical Technology 2011 (2011) 510186.

[3] Y. Terada, S. Yoshida, O. Takeuchi, and H. Shigekawa: Nature Photonics 4 (2010) 869.

[4] S. Yoshida, Y. Terada, R. Oshima, O. Takeuchi, and H. Shigekawa: Nanoscale 4 (2012) 757.

[5] S. Yoshida, Y. Terada, M. Yokota, O. Takeuchi, H. Oigawa, and H. Shigekawa: The European Physical Journal Special Topics 222 (2013) 1161-1175.

[6] S. Yoshida, M. Yokota, O. Takeuchi, and H. Shigekawa: Appl. Phys. Exp. 6, (2013) 016601.

[7] S. Yoshida, O. Takeuchi, H. Oigawa, and H. Shigekawa: Appl. Phys. Exp. 6, (2013) 032401.

[8] M. Yokota, S. Yoshida, Y. Mera, O. Takeuchi, H. Oigawa, and H. Shigekawa, Nanoscale 5 (2013) 9170.

[9] S. Yoshida, Y. Aizawa, Z. Wang, R. Oshima, Y. Mera, E. Matsuyama, H. Oigawa, O. Takeuchi, and H. Shigekawa: Nature Nanotechnology 9 (2014) 588.

[10] H. Shigekawa, S. Yoshida and O. Takeuchi: Nature Photonics 8 (2014) 815.
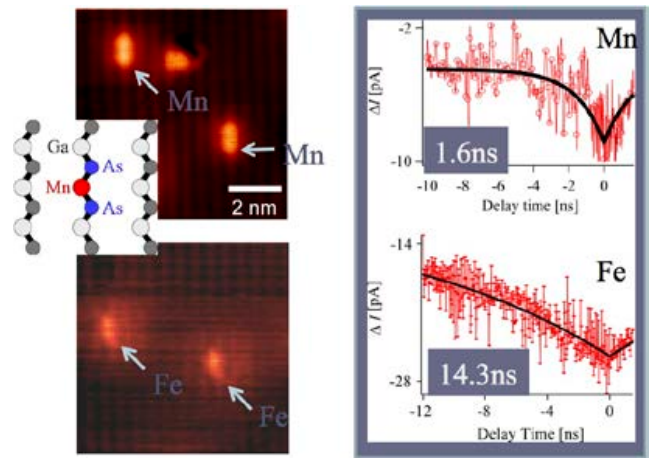

Fig. 1
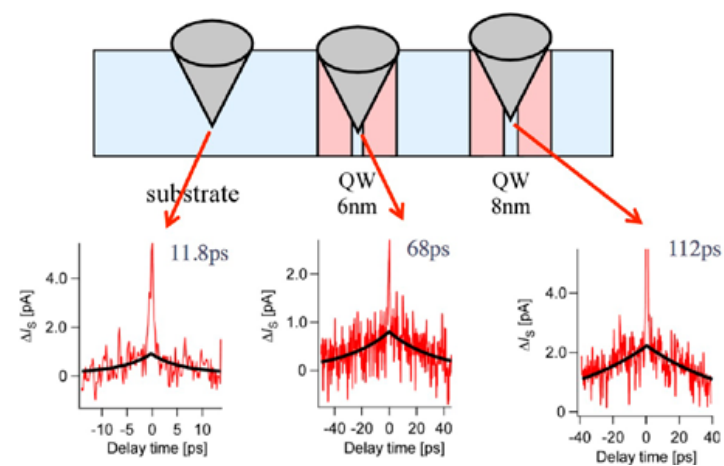

Fig. 2

Figure 1. Time-resolved spectra of hole capture process at single $\mathrm{Mn} / \mathrm{Fe}$ atoms deposited on a GaAs(110) surface [5, 7].

Figure 2. Time-resolved spectra of spin dynamics in single GaAs/AlGaAs quantum wells [9].

(a)

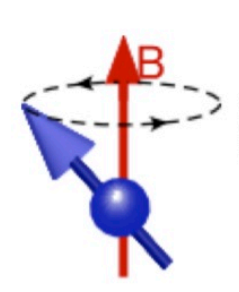

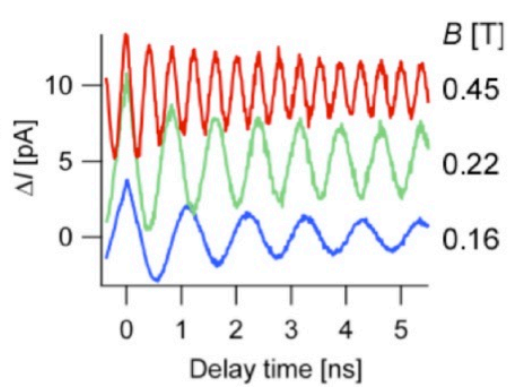

Fig. 3 (b)

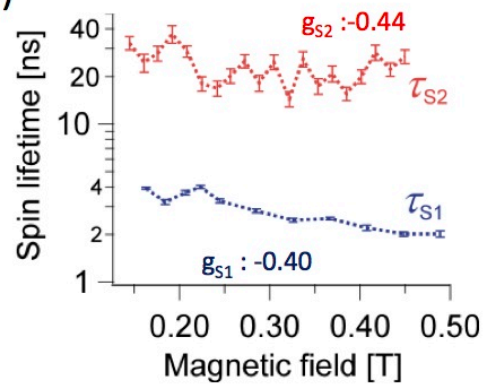

Figure 3. (a) Precession of optically oriented spins in GaAs measured with the newly developed optical pump-probe STM, and (b) local g factors obtained from the full series of the data [9]. 